\title{
Carbonized Aramid Fiber as the Adsorbent for In-Tube Solid-Phase Microextraction to Detect Estrogens in Water Samples
}

\author{
Xiaoxiao Zhu $\mathbb{D}^{1,2}$ Yijun Zhang $\mathbb{D},{ }^{1,2}$ Pengfei Liu, ${ }^{2}$ Xiuzhi Bai, ${ }^{2}$ Na Chen, \\ and Yuping Zhang ${ }^{1,2}$ \\ ${ }^{1}$ College of Chemistry, Zhengzhou University, Zhengzhou 450001, Henan, China \\ ${ }^{2}$ College of Chemistry and Chemical Engineering, Henan Institute of Science and Technology, Xinxiang 453003, Henan, China
}

Correspondence should be addressed to Yijun Zhang; zhangyijun@hist.edu.cn

Received 12 March 2021; Revised 20 April 2021; Accepted 30 April 2021; Published 26 May 2021

Academic Editor: Ajaya Kumar Singh

Copyright ( 2021 Xiaoxiao Zhu et al. This is an open access article distributed under the Creative Commons Attribution License, which permits unrestricted use, distribution, and reproduction in any medium, provided the original work is properly cited.

Carbonized aramid fiber was prepared as a new type of adsorbent for in-tube solid-phase microextraction. The surface structure, chemical composition, and graphitization degree of the resulted fiber was determined and characterized by scanning electron microscopy, X-ray photoelectron spectroscopy, and Raman spectrometry. The prepared fiber was packed in a stainless-steel tube instead of the sample loop of a six-port and tested for the extraction of five environmental estrogen hormones coupled with highperformance liquid chromatography. Several parameters affecting the estrogens' extraction including the sampling volume, sampling rate, $\mathrm{NaCl}$ content, and desorption time were investigated in detail. The extraction tube with carbonized aramid fiber exhibited remarkable extraction performance towards five estrogen targets. The analysis method was established, and it exhibited a wide linear range $(0.5-10.0 \mu \mathrm{g} / \mathrm{L})$ with good linearity (correlation coefficient $\geq 0.9906)$, low limits of detection $(0.011-0.13 \mu \mathrm{g} / \mathrm{L})$, and high enrichment factors (178-1335) for the five analytes. Relative standard deviations $(n=3)$ for intraday $(\leq 4.8 \%)$ and interday $(\leq 4.0 \%)$ tests indicated that the extraction material had satisfactory repeatability. Bisphenol A released from a polycarbonate (PC) bottle was quantitatively detected with a concentration of $8.3 \mu \mathrm{g} / \mathrm{L}$. The relative recoveries spiked at 5 and $10 \mu \mathrm{g} / \mathrm{L}$ were investigated, and the results were in the range of $74.3-121 \%$ for real water samples.

\section{Introduction}

There is growing interest in the presence of micropollutants in the environment that can interfere with the endocrine system and affect the health, growth, and reproduction of humans and animals $[1,2]$. These substances are known as endocrine-damaging chemicals (EDCs) and are related to various human diseases (such as prostate cancer, reproductive tract diseases, low sperm count, and breast cancer) [3-5]. As a typical endocrine disruptor, estrogen has been detected in sewage, surface water, and even drinking water in different countries [6, 7]. For example, bisphenol A (BPA) as a prototypical EDC is an estrogenic high-production chemical used primarily as a monomer to produce epoxy resins and polycarbonate. It is now well known that there is ubiquitous human exposure to BPA [8]. For this reason, there is an urgent need to investigate the contamination of estrogens in the natural environment.
The analytes from the complex matrices are often present at low concentrations. It requires the development of sensitive and specific methods for the determination of target analytes [9]. Solid-phase microextraction (SPME) was firstly introduced by Arthur and Pawliszyn in the early 1990s [10]. SPME is a simple and convenient sample preparation technique that has enabled automation, miniaturization, and high-throughput performance [11-18]. In-tube SPME is a novel SPME technique that is easy to operate, solvent free, and cost effective [19-22]. Coupling with high-performance liquid chromatography (HPLC), in-tube SPME can be successfully applied to separate and detect the target analytes in liquid samples online [23-26]. Nowadays, the most important task for in-tube SPME is to develop efficient extraction materials. Many reports use different materials for in-tube SPME to extract environmental organic pollutants and obtained good extraction efficiency, for example, cotton fiber, basalt fiber, polypropylene hollow fiber, and coatings 
based on them [27-30]. Although many materials have been developed as sorbents for the SPME, their synthesis processes are more complex, and the use of organic solvent is too much in a way. Thereby, it is more meaningful to develop a cheap, simple, and green sorbent used as the support for intube SPME.

Recently, aramid fiber, one of the representatives in the high-performance engineered fibers family, has attracted wide attention due to its specific properties with soft texture, low density, high strength, and excellent thermal stability. It has been widely used in the field of aircraft, aerospace, tanks, missile, and so on [31, 32]. Poly-p-phenylene terephthalamide (PPTA) fiber is the most common type of aramid fiber. It is also called para-aramid, and its main chain of the macromolecule is a linear molecular structure formed alternately by amide bonds and benzene rings. If it is chosen as a sorbent for in-tube SPME, the multiple interactions including $\pi-\pi$ stacking, electrostatic interaction, and hydrogen bond may occur between its surfaces and the extracted target compounds of estrogenic hormones.

Based on these considerations, aramid fiber and its carbonized fiber under high temperature were initially selected as the sorbents for in-tube SPME. Both fibers were filled in the stainless-steel tubes to make the extraction tubes. The extraction tube was connected to the injection valve of HPLC, respectively, and an online analysis system was constructed. Five common estrogenic hormones were selected as the model analytes to investigate the extraction performance using two kinds of fibers. The online analytical method was established and applied to the determination of five estrogens in two real samples using the optimal extraction and desorption conditions.

\section{Materials and Methods}

2.1. Materials and Reagents. Aramid fiber was obtained from Japan Toho Chemical Industry Co., Ltd. Methanol and acetonitrile were HPLC grade and purchased from Tianjin Damao Reagent Company (Tianjin, China). Five kinds of natural and synthetic estrogens including BPA (>99.0\%), ethinylestradiol (>98.0\%), diethylstilbestrol (>99.0\%), hexestrol (>98.0\%), and estrone (>98.0\%) were purchased from Shanghai Aladdin Biochemical Co., Ltd. (Shanghai, China). Their chemical structures are shown in Figure 1. Ultrapure water $\left(18.25 \mathrm{M} \Omega \mathrm{cm}, 25^{\circ} \mathrm{C}\right)$ was used for the whole experiment. The polycarbonate (PC) bottle was purchased from a local supermarket. The mobile phase was filtered through a $0.2 \mu \mathrm{m}$ organic membrane. Water/acetonitrile $(53: 47, \mathrm{v} / \mathrm{v})$ was used as the mobile phase to elute estrogens at the detection wavelength of $202 \mathrm{~nm}$.

2.2. Apparatus. The Agilent 1260 quaternary pump was used as a sample pump to transport sample solutions. Analytes were detected by using the Agilent 1220 HPLC system equipped with a ZORBAX SB-C18 column $(150 \times 4.6 \mathrm{~mm}$ i.d., $5 \mu \mathrm{m}$, Agilent Technologies) and a variable wavelength detector (VWD). Aramid fiber and carbonized aramid fiber were characterized by SEM (quanta 200 environmental scanning electron microscope), X-ray photoelectron spectra (XPS, Thermo ESCALAB 250XI, USA), and Raman spectrometry (NEXUS 670, Horiba Jobin Yvon, France). Aramid fiber was carbonized by using a high-temperature tube furnace (OTF-1200X-S, Hefei Kejing Material Technology Co., Ltd., China).

2.3. Standard Solution and Real Samples. The stock solution $(100 \mathrm{mg} / \mathrm{L})$ containing five estrogens was prepared with methanol and stored at $4^{\circ} \mathrm{C}$.

The working solution was prepared daily from by dilution of the stock solution with pure water to $10 \mu \mathrm{g} / \mathrm{L}$. The boiled ultrapure water was filled into a new $1 \mathrm{~L}$ PC bottle and cooled to room temperature. Both the cooled water in the PC bottle and tap water taken from the laboratory were selected as real samples for the evaluation. All water samples were filtered with a $0.45 \mu \mathrm{m}$ water membrane before chromatographic analysis.

2.4. Preparation of the Extraction Tube. The aramid fiber was ultrasonically cleaned by acetonitrile for $20 \mathrm{~min}$, repeated 3 times, and then, dried. After drying, it was placed in a hightemperature tube furnace with nitrogen protection. The carbonized temperature is investigated in the range of $500^{\circ} \mathrm{C}-600^{\circ} \mathrm{C}$

for $6 \mathrm{~h}$. Afterwards, 8 strands of carbonized aramid fiber with a length of $30 \mathrm{~cm}$ and a weight of $40 \mathrm{mg}$ were filled into stainless-steel tubes $(0.75 \mathrm{~mm}$ i.d. and $1 / 16$ inch o.d.), respectively. Aramid fiber with the same length and weight without carbonization was also filled into the extraction tube for comparison.

2.5. Extraction and Analysis Procedure. As shown in Figure 2, the extraction tube was connected to the loop position on the six-port valve of the Agilent 1220 HPLC system. The six-port valve was switched between load and inject states to perform the extraction and desorption processes. In the load state, the sample solution is transported through the extraction tube via the Agilent 1260 quaternary pump and the target analytes are adsorbed by the selected aramid fiber. In the injection state, the mobile phase $(1.00 \mathrm{~mL} / \mathrm{min})$ flows through the extraction tube and the absorbed target compounds are desorbed and are detected consequently. The extraction and the desorption can be completed by the switching between load and inject modes.

In order to calculate the enrichment factor (EF) values more accurately, a series of standard solutions were prepared in the range of $0-100 \mathrm{mg} / \mathrm{L}$. After $20 \mu \mathrm{L}$ standard solutions with different concentrations of $0,0.25,0.5,1,2.5,5,10,25$, and $100 \mathrm{mg} / \mathrm{L}$ were injected using the sample loop, respectively, the calibration curve with a slope of $k_{1}$ was then obtained according to the peak area vs. concentration. Moreover, another series of standard solutions with a constant sampling volume of $50 \mathrm{~mL}$ and different concentrations of $0,0.5,1,2,5$, and $10 \mu \mathrm{g} / \mathrm{L}$ were prepared. After they are injected and detected by using an in-tube SPME-HPLC, the calibration curve was then obtained with a slope of $k_{2}$. The final EF can be calculated according the following equation: $\mathrm{EF}=k_{2} / k_{1}$. 




(a)



(b)

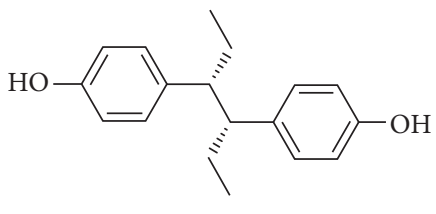

(c)<smiles>[CH][C@@]12CC[C@@H]3c4ccc(O)cc4CC[C@H]3[C@@H]1CC[C@@]2(O)C#C</smiles>

(d)<smiles>C[C@]12CC[C@H]3c4ccc(O)cc4CC[C@H]3[C@@H]1CCC2=O</smiles>

(e)

Figure 1: Chemical structures of natural and synthetic estrogens used in this study. (a) Bisphenol A. (b) Diethylstilbestrol. (c) Hexestrol. (d) Ethinyl estradiol. (e) Estrone.

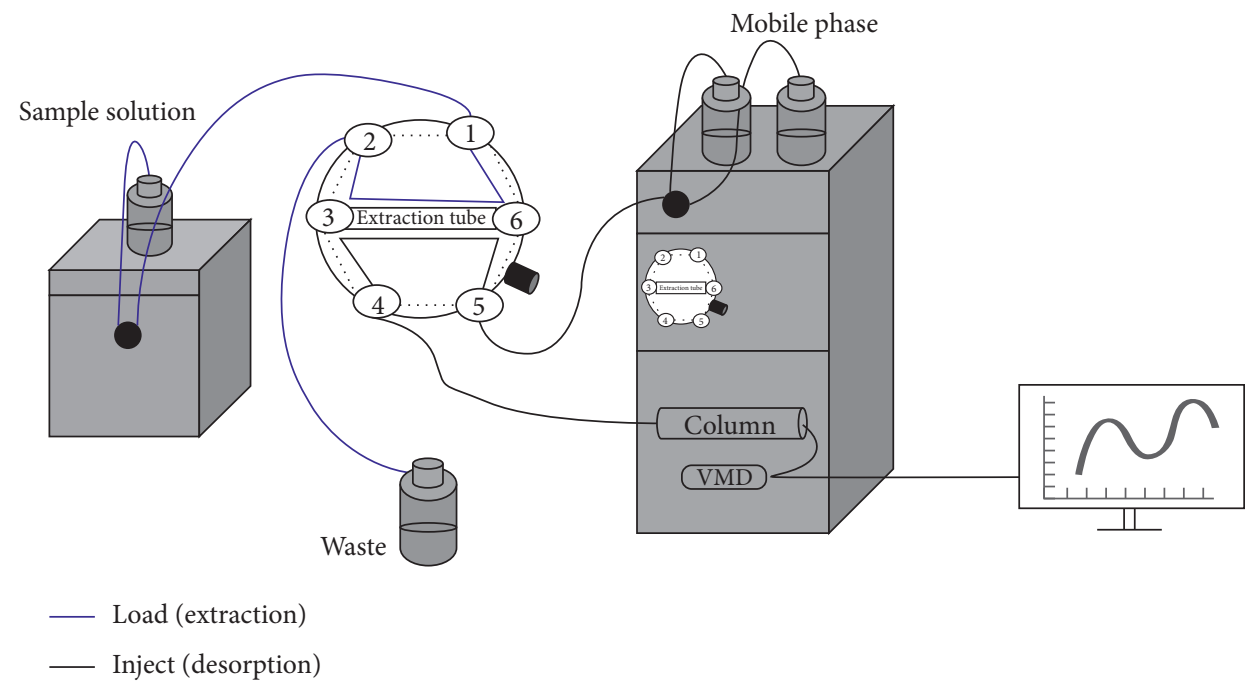

FIGURE 2: Construction of an online in-tube SPME-HPLC online system.

\section{Results and Discussion}

3.1. Characterization. The morphological properties of aramid fiber and carbonized aramid fiber were characterized by SEM. As can be seen from Figure 3, the aramid fiber had a smooth surface with few grooves. After carbonization at high temperature, some small holes appeared, and the increasement of roughness led to an increase of adsorption sites in extraction.

Raman characterizations were also carried out for the aramid fiber and its carbonized aramid fiber. As shown in Figure 4, there was a G-band, which corresponded to the inplane tangential vibration of graphite-like carbon about $1580 \mathrm{~cm}^{-1}$ and a D-band associated with disordered carbon around $1340 \mathrm{~cm}^{-1}$ for the carbonized aramid fiber [33]. In contrast, no obvious characteristic peak was found on the aramid fiber. The intensity ratio of D-band to G-band was around 1.17, indicating that high temperature caused its partial carbonization with more graphitized carbon atoms appeared on its surface.
As shown in Figure 5, the X-ray photoelectron spectroscopy (XPS) spectrum indicated the carbonized aramid fiber mainly contained elements of carbon and oxygen. The atomic percent of $\mathrm{C}$ and $\mathrm{O}$ was 69.4 and $17.6 \%$, respectively. The C 1 s XPS spectrum can be fitted into three components, and these peaks at 284.1, 284.5, and $287.7 \mathrm{eV}$ correspond to the carbon bonds of $\mathrm{C}-\mathrm{C}$, $\mathrm{C}-\mathrm{OH}$, and $\mathrm{C}=\mathrm{O}$. The results showed that amorphous carbon was formed in situ on the surface of the carbonized aramid fiber.

3.2. Optimization of Extraction Conditions. The treatment temperature directly affects the carbonization degree of aramid fiber, which decides its extraction performance. In our experiments, the carbonization temperature was investigated in the range of $500-600^{\circ} \mathrm{C}$

with a constant carbonization time of $6 \mathrm{~h}$. Under the optimal conditions, the extraction efficiency to five estrogens was comparatively evaluated for the 


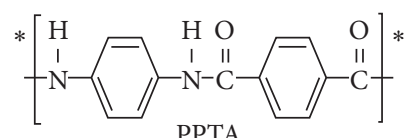

PPTA

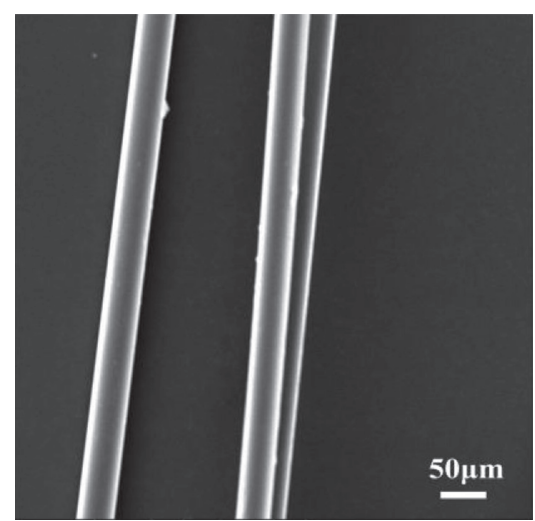

(b)

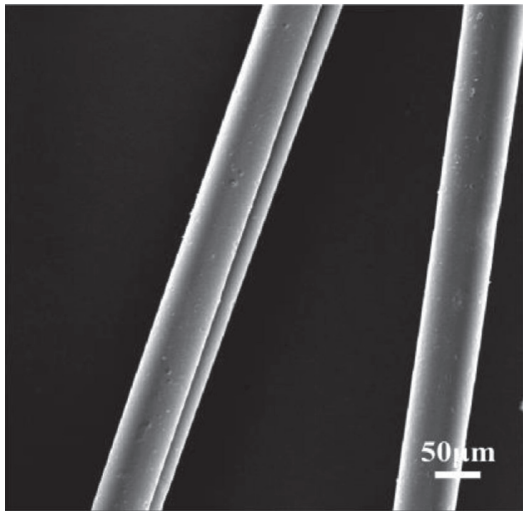

(e)

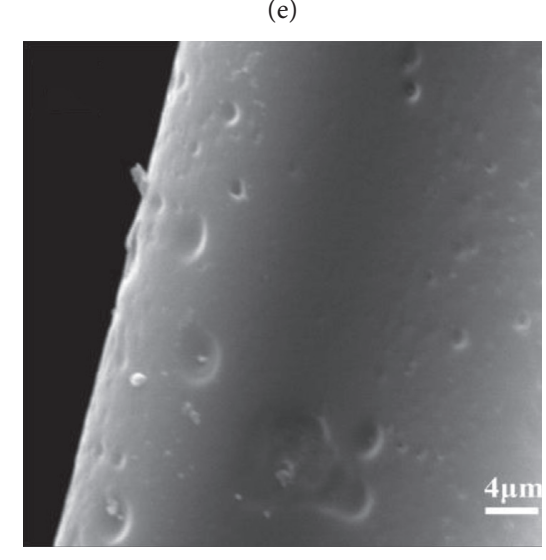

$(\mathrm{g})$$$
\text { . }
$$


(c)

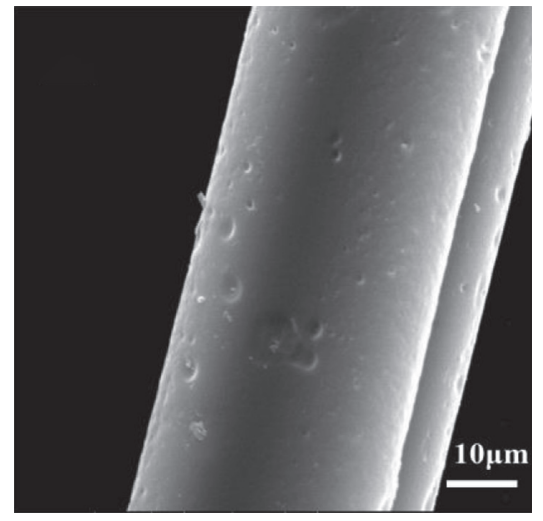

(f)

(d)

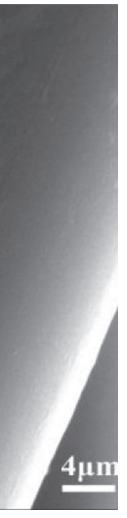

\section{(g)}

FIgURE 3: The chemical structure of PPTA (a); SEM micrographs of aramid fiber before (b, c, and d) and after being carbonized (e, f, and g).

carbonized aramid fiber under different temperatures and the pristine aramid fiber. As shown in Figure 6, the pristine aramid fiber and the carbonized aramid fiber at $570^{\circ} \mathrm{C}$ and $600^{\circ} \mathrm{C}$ presented the lowest extraction efficiency. When the carbonization temperature was $500^{\circ} \mathrm{C}$ and $550^{\circ} \mathrm{C}$, the highest extraction efficiency of five estrogens was obtained. It can be seen from Table 1 that the largest peak areas of BPA, ethynylestradiol, diethylstilbestrol, and hexestrol were achieved at $500^{\circ} \mathrm{C}$, but the peak area of estrone was the highest at $550^{\circ} \mathrm{C}$.
It was probably attributed to that carbonization at suitable temperature promoted the structural regularity and stability of carbon atoms on the surface of aramid fiber. In this situation, more adsorption sites were created with higher extraction efficiency for the target compounds; aramid fiber carbonized at $500^{\circ} \mathrm{C}$ was selected as the extraction phase for next in-tube SPMEHPLC.

When the peak area tends to be constant with the increase of the sample volume, the extraction reaches equilibrium and the largest extraction efficiency will be obtained. 


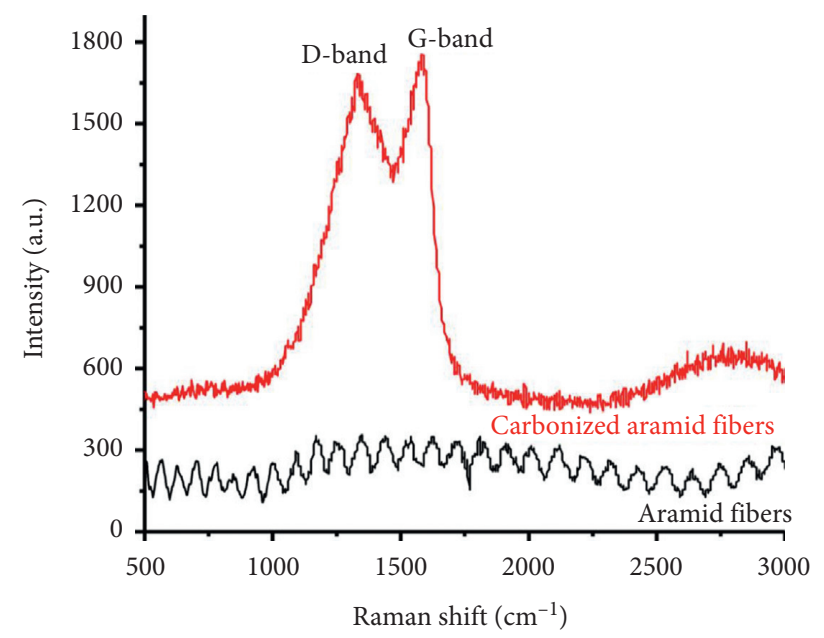

Figure 4: Raman spectra of aramid fiber and carbonized aramid fiber.

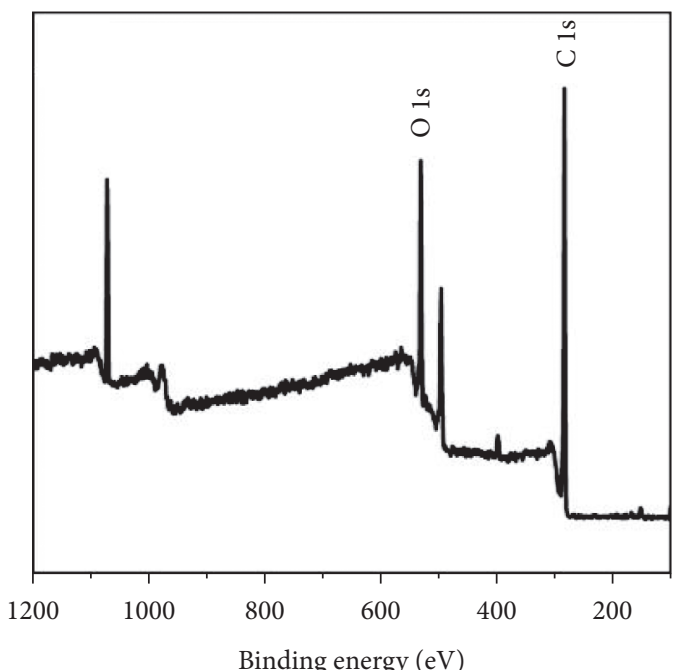

(a)

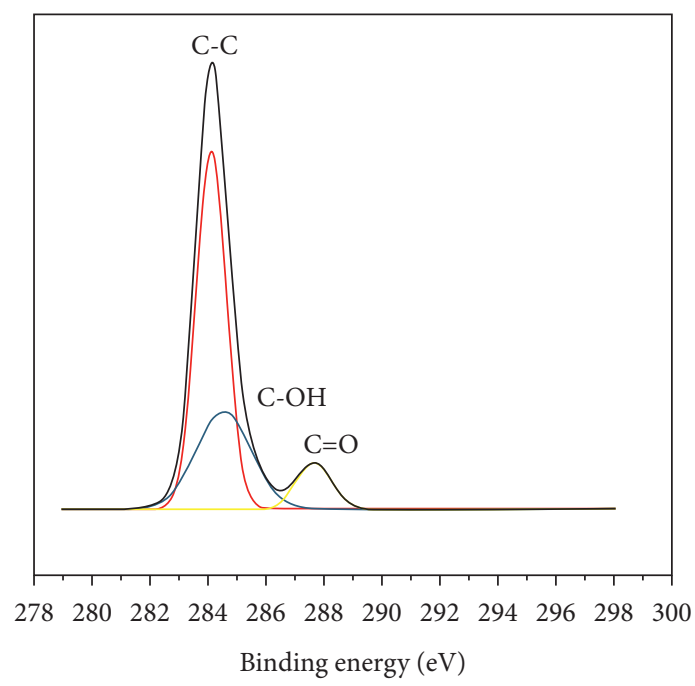

(b)

Figure 5: XPS survey (a) and the C 1s core level XPS spectrum of the carbonized aramid fiber (b).

However, excessive sampling volume may result in a waste of extraction time and affect extraction efficiency. As shown in Figure $7(\mathrm{a})$, peak areas of the analytes increased with the change of sampling volume from 20 to $80 \mathrm{~mL}$, especially for diethylstilbestrol. The increase of peak areas was unremarkable for other compounds when the sampling volume was more than $50 \mathrm{~mL}$. Compromising the extraction efficiency and time, $50 \mathrm{~mL}$ was selected as the optimal sampling volume.

Sampling rate, as one of the optimized conditions, has a significant impact on extraction efficiency and extraction time. After the same sampling volume of $50 \mathrm{~mL}$ was loaded, the sampling rate was investigated in the range of $0.50-2.00 \mathrm{~mL} / \mathrm{min}$. It can be seen from Figure $7(\mathrm{~b})$ that the peak areas of the five analytes changed little with increasing the sampling rate from 0.50 to $1.00 \mathrm{~mL} / \mathrm{min}$. While the sampling rate increases beyond $1.00 \mathrm{~mL} / \mathrm{min}$, the upward trend can be observed easily. In order to obtain better extraction efficiency and quick analysis, $2.00 \mathrm{~mL} / \mathrm{min}$ was chosen as the optimal sampling rate.

The addition of inorganic salts such as $\mathrm{NaCl}$ to the water sample can increase the ionic strength of the solution, effectively reduce the dissolution of organic compounds, and improve the extraction efficiency of SPME. On the other hand, it will increase the solution's viscosity and reduce the diffusion rate of organic matter. Herein, $\mathrm{NaCl}$ in the concentration range of $0-2.0 \%(\mathrm{w} / \mathrm{w})$ was added in the sample solutions for the investigation. As shown in Figure 7(c), with the increase of $\mathrm{NaCl}$ content, each sample's peak area gradually decreased, indicating that the best extraction efficiency was obtained for the sample without $\mathrm{NaCl}$. Therefore, the addition of $\mathrm{NaCl}$ was not considered in the subsequent experiments.

After the extraction was completed, water/acetonitrile $(53: 47, \mathrm{v} / \mathrm{v})$ as the mobile phase flowed through the 


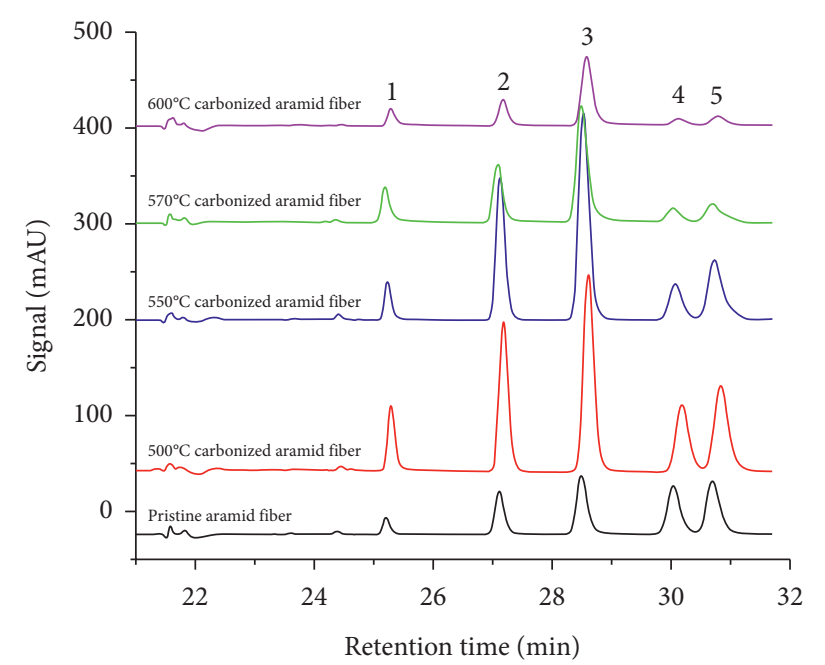

Figure 6: Chromatograms of aramid fiber and aramid fiber carbonized at different temperatures. Peaks: (1) bisphenol A, (2) ethynylestradiol, (3) diethylstilbestrol, (4) hexestrol, and (5) estrone. Conditions: sampling volume, $50 \mathrm{~mL}$; sampling rate, $2.00 \mathrm{~mL} / \mathrm{min}$; and desorption time, $2.0 \mathrm{~min}$. Mobile phase: water/acetonitrile (53:47, v/v); detection wavelength, $202 \mathrm{~nm}$; and room temperature.

TABle 1: Comparison of enrichment capacity of aramid fiber and aramid fibers carbonized at different temperatures.

\begin{tabular}{|c|c|c|c|c|c|}
\hline \multirow[b]{2}{*}{ Analytes } & \multicolumn{5}{|c|}{ Peak areas $(\mathrm{mAU} * \mathrm{~S})$} \\
\hline & Pristine aramid fiber & $\begin{array}{c}\text { Aramid fiber } \\
\text { carbonized at } 500^{\circ} \mathrm{C}\end{array}$ & $\begin{array}{c}\text { Aramid fiber } \\
\text { carbonized at } 550^{\circ} \mathrm{C}\end{array}$ & $\begin{array}{c}\text { Aramid fiber } \\
\text { carbonized at } 570^{\circ} \mathrm{C}\end{array}$ & $\begin{array}{c}\text { Aramid fiber } \\
\text { carbonized at } 600^{\circ} \mathrm{C}\end{array}$ \\
\hline Bisphenol A & 168 & 603 & 361 & 448 & 226 \\
\hline Ethynyl estradiol & 626 & 1696 & 1627 & 680 & 312 \\
\hline Diethylstilbestrol & 1001 & 2643 & 2781 & 1712 & 1041 \\
\hline Hexestrol & 916 & 936 & 605 & 219 & 119 \\
\hline Estrone & 1092 & 1542 & 1156 & 351 & 164 \\
\hline
\end{tabular}

extraction tube at $1.00 \mathrm{~mL} / \mathrm{min}$. The analytes absorbed by the support in the extraction tube were eluted, and the desorption process was carried out. Adequate desorption time ensured the full desorption of all extracted analytes and reduced the impact of residual analytes on the next extraction. The effect of desorption time was investigated in the range of $0.20-2.00 \mathrm{~min}$. As shown in Figure $7(\mathrm{~d})$, five estrogens' peak areas exhibited an upward trend with the increasement of desorption time; thus $2.00 \mathrm{~min}$ was selected as the optimal desorption time.

3.3. Method Evaluation and Application to Real Samples. Under optimized conditions, a series of parameters including linear range, correlation coefficient $\left(R^{2}\right)$, interday repeatability, intraday repeatability, limits of detection (LODs), and limits of quantification (LOQs) were investigated to validate the online in-tube SPME-HPLC method. As shown in Table 2, in the selected concentration range of 0.5-10.0 $\mu \mathrm{g} / \mathrm{L}$, all analytes exhibited satisfactory correlation coefficients $\left(R^{2}\right)$ of $0.9915,0.9939,0.9962,0.9906$, and 0.9941, for bisphenol A, ethynylestradiol, diethylstilbestrol, hexestrol, and estrone, respectively. LODs $(\mathrm{S} / \mathrm{N}=3)$ and LOQs $(\mathrm{S} / \mathrm{N}=10)$ were within the ranges of $0.017-0.13 \mu \mathrm{g} / \mathrm{L}$ and $0.038-0.45 \mu \mathrm{g} / \mathrm{L}$, respectively. Three parallel tests were performed in one day to investigate the intraday extraction repeatability, and their relative standard deviations $(n=3)$ were between $0.6 \%$ and $4.8 \%$. Under the same conditions, an experiment was performed once a day for three days to get the interday extraction repeatability, and the relative standard deviation (RSDs, $n=3$ ) of the peak area was less than $4.0 \%$. The extraction material has good extraction repeatability. In addition, three extraction devices prepared in different batches $(n=3)$ were chosen to explore the reproducibility of peak areas, and the satisfactory results were obtained with their RSDs in the range of 1.1-9.2\%. The EFs (BPA, ethynylestradiol, diethylstilbestrol, hexestrol, and estrone) were equal to the ratio of the slope of the standard curve before (corresponding $k_{1}=0.19,0.14,0.13,0.16,0.15$ ) and after (corresponding $k_{2}=34.4,101.3,174.9,101.5,109.5$ ) enrichment. Under optimized conditions, each estrogen component on the extraction tube was enriched effectively with their values in the range of 178-1335.

The developed method was compared with other reported methods for the determination for estrogens, including extraction materials, extraction time, enrichment factors, and recoveries. Relative data are presented in Table 3 . Although the recoveries obtained by our developed method were close or comparable to other work [34-39], the LOD value was better than that of other methods except the 

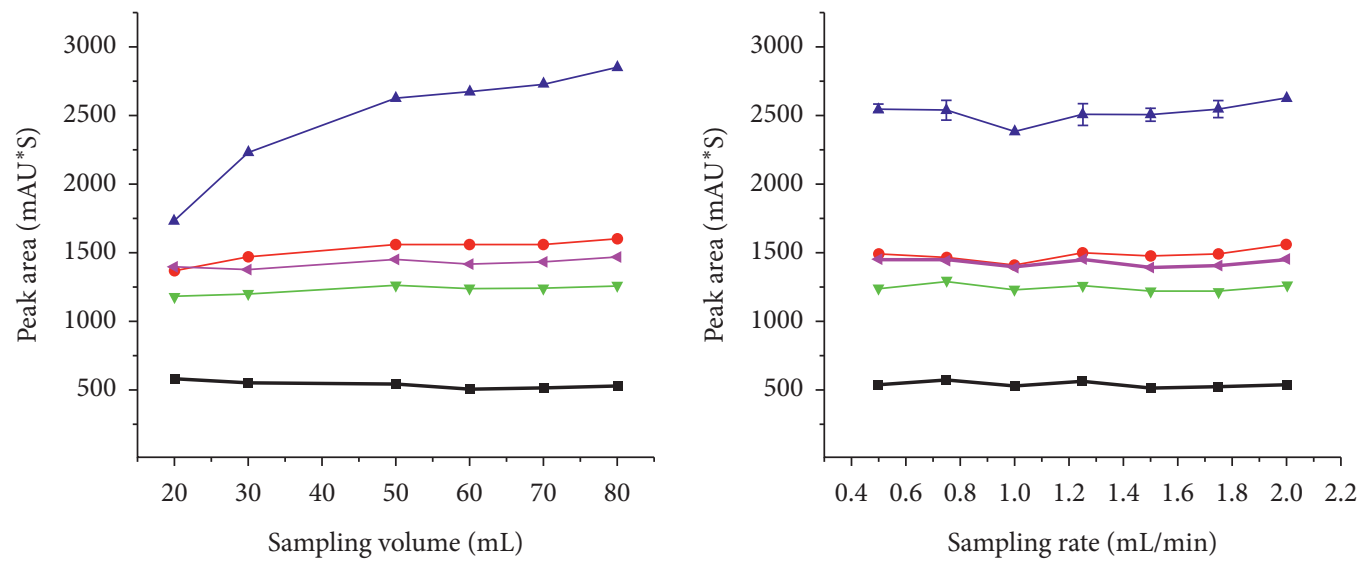

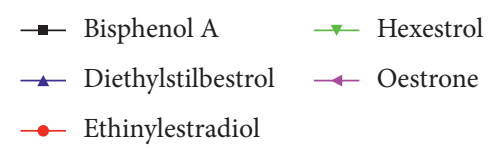

(a)

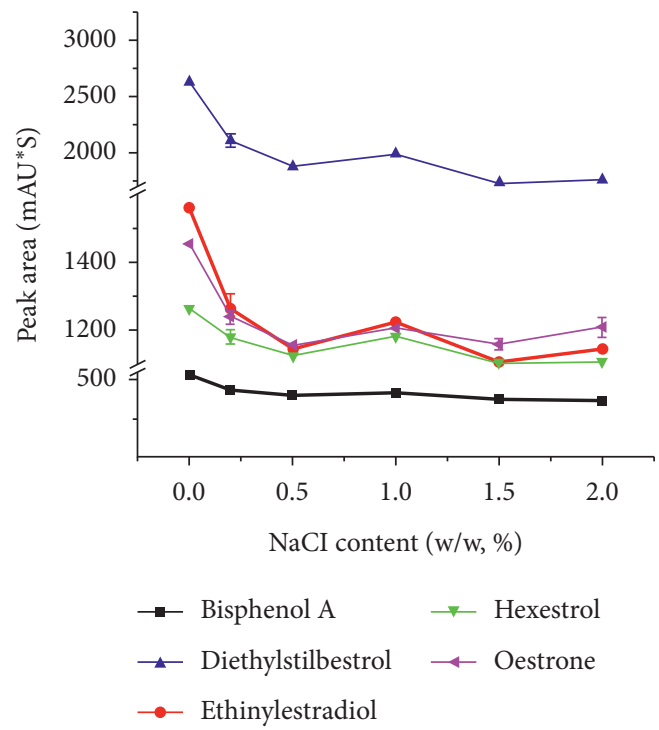

(c)

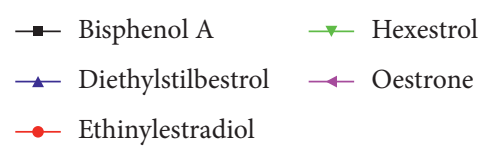

(b)

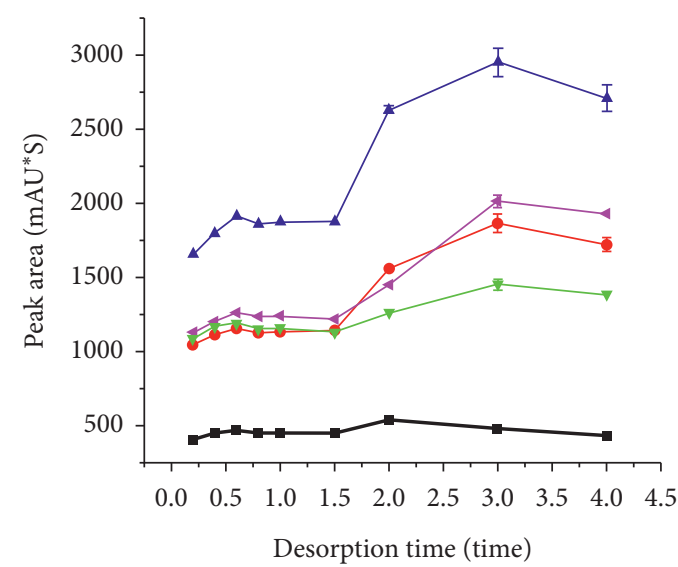

$\rightarrow$ Bisphenol A $\quad \longleftarrow$ Hexestrol
$\longleftarrow$ Diethylstilbestrol $\longleftarrow$ Oestrone
$\multimap$ Ethinylestradiol

(d)

FIgURE 7: The optimization of extraction conditions including (a) sampling volume, (b) sampling rate, (c) $\mathrm{NaCl}$ content, and (d) desorption time. Condition: concentration of estrogens, $10 \mu \mathrm{g} / \mathrm{L}$. Other conditions are the same as in Figure 6.

TABLE 2: Analytical performances of the in-tube SPME-HPLC method for the detection of five estrogens.

\begin{tabular}{|c|c|c|c|c|c|c|c|c|}
\hline \multirow[t]{2}{*}{ Analytes } & \multirow[t]{2}{*}{ LODs $(\mu \mathrm{g} / \mathrm{L})$} & \multirow[t]{2}{*}{$\begin{array}{l}\text { LOQs } \\
(\mu \mathrm{g} / \mathrm{L})\end{array}$} & \multirow[t]{2}{*}{$\begin{array}{l}\text { Linear ranges } \\
\qquad(\mu \mathrm{g} / \mathrm{L})\end{array}$} & \multirow[t]{2}{*}{$r^{2 \mathrm{a}}$} & \multirow[t]{2}{*}{ Enrichment factors ${ }^{\mathrm{b}}$} & \multicolumn{2}{|c|}{$\begin{array}{c}\text { Extraction } \\
\text { repeatability }(n=3 \text {, } \\
\text { RSD \% })^{c}\end{array}$} & \multirow[t]{2}{*}{$\begin{array}{l}\text { Preparation repeatability } \\
\qquad(n=3, \mathrm{RSD} \%)^{\mathrm{d}}\end{array}$} \\
\hline & & & & & & Intraday & Interday & \\
\hline Bisphenol A & 0.027 & 0.089 & $0.5-10.0$ & 0.9915 & 178 & 4.8 & 1.8 & 2.3 \\
\hline Ethynyl estradiol & 0.017 & 0.055 & $0.5-10.0$ & 0.9939 & 734 & 0.6 & 1.1 & 1.1 \\
\hline Diethylstilbestrol & 0.011 & 0.038 & $0.5-10.0$ & 0.9962 & 1335 & 1.1 & 1.0 & 1.9 \\
\hline Hexestrol & 0.13 & 0.45 & $0.5-10.0$ & 0.9906 & 634 & 2.3 & 2.1 & 9.2 \\
\hline Estrone & 0.061 & 0.20 & $0.5-10.0$ & 0.9941 & 750 & 1.7 & 4.0 & 3.9 \\
\hline
\end{tabular}

${ }^{a}$ Calibration level: $n=3$. ${ }^{\mathrm{b}}$ Enrichment factors were obtained by extracting $10 \mu \mathrm{g} / \mathrm{L}$ standard solution of estrogen under the optimized conditions. ${ }^{\mathrm{c}}$ Extraction repeatability was investigated by extracting standard solution spiked at $10 \mu \mathrm{g} / \mathrm{L}$ of estrogens three times. ${ }^{\mathrm{d}}$ Preparation repeatability was investigated by extracting standard solution spiked at $10 \mu \mathrm{g} / \mathrm{L}$ of estrogens with three extraction tubes. 
TABLE 3: Comparison with other reported methods for the determination of estrogens.

\begin{tabular}{|c|c|c|c|c|c|c|}
\hline Analysis methods & $\begin{array}{l}\text { Extraction } \\
\text { materials }\end{array}$ & $\begin{array}{l}\text { LOD } \\
(\mu \mathrm{g} / \mathrm{L})\end{array}$ & EFs & $\begin{array}{l}\text { Extraction } \\
\text { time (min) }\end{array}$ & $\begin{array}{l}\text { Recoveries } \\
(\%)\end{array}$ & Ref. \\
\hline IT-SPME-HPLC-VWD & Carbonized aramid fibers & $0.011-0.130$ & $178-1335$ & 32 & $74.3-121.1$ & This work \\
\hline HF-PIL-capsules-SPME & Hollow fiber membrane-coated PIL & $1.000-2.000$ & $5-200$ & 40 & - & {$[34]$} \\
\hline SPME-HPLC-UV & Molecularly imprinted polymer & $2.210-2.390$ & - & 38 & $80.0-94.0$ & {$[35]$} \\
\hline $\begin{array}{l}\text { AIED/SCSE-HPLC/ } \\
\text { DAD }\end{array}$ & Polymer ionic liquid & $0.025-0.057$ & $71-97$ & 150 & $82.9-99.4$ & {$[36]$} \\
\hline CPE-HPLC/DAD & Tergitol TMN-6 & $0.100-0.200$ & $66.51-94.34$ & 60 & $85.9-104.0$ & [37] \\
\hline HPLC-FLD-UV & NH2-MIL-53 (Al)-polymer monolith & $0.002-0.040$ & $289-293$ & - & $75.1-102.0$ & {$[38]$} \\
\hline SPE-HPLC-DAD & Molecularly imprinted polymers & $8.71-9.54$ & - & - & $69.0-82.2$ & [39] \\
\hline
\end{tabular}

TABLe 4: Analytical results and recoveries of five estrogens in two real samples.

\begin{tabular}{|c|c|c|c|c|c|}
\hline Analytes & Bisphenol A & Ethynylestradiol & Diethylstilbestrol & Hexestrol & Estrone \\
\hline Water in the PC bottle $(\mu \mathrm{g} / \mathrm{L})$ & $8.3 \pm 0.2$ & Not detected & Not detected & Not detected & Not detected \\
\hline Recovery $(5 \mu \mathrm{g} / \mathrm{L}, \%)$ & $113.9 \pm 7.0$ & $100.4 \pm 1.6$ & $89.3 \pm 2.2$ & $119.0 \pm 4.0$ & $121.1 \pm 3.1$ \\
\hline Recovery $(10 \mu \mathrm{g} / \mathrm{L}, \%)$ & $84.3 \pm 1.6$ & $75.0 \pm 1.7$ & $89.7 \pm 0.7$ & $108.6 \pm 5.5$ & $120.3 \pm 8.1$ \\
\hline Tap water $(\mu \mathrm{g} / \mathrm{L})$ & Not detected & Not detected & Not detected & Not detected & Not detected \\
\hline Recovery $(5 \mu \mathrm{g} / \mathrm{L}, \%)$ & $112.2 \pm 4.0$ & $89.4 \pm 0.2$ & $86.6 \pm 1.6$ & $120.7 \pm 1.5$ & $102.4 \pm 2.0$ \\
\hline Recovery $(10 \mu \mathrm{g} / \mathrm{L}, \%)$ & $87.7 \pm 5.3$ & $76.4 \pm 0.6$ & $74.6 \pm 0.8$ & $107.8 \pm 1.9$ & $87.5 \pm 1.6$ \\
\hline
\end{tabular}

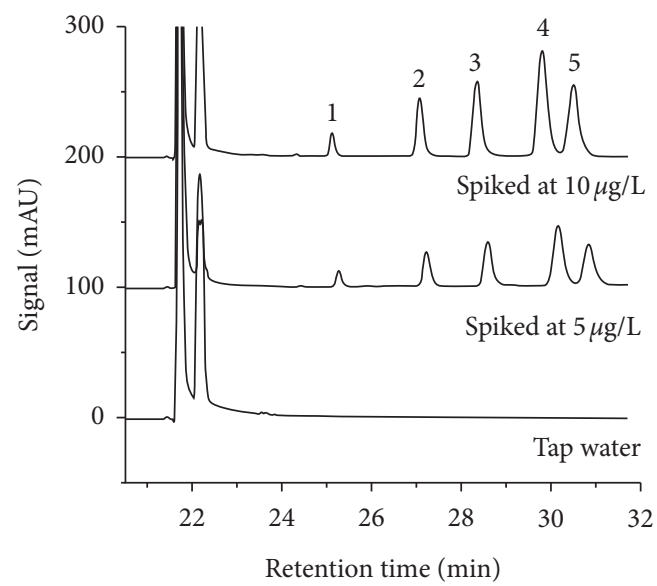

(a)



(b)

Figure 8: HPLC chromatograms of (a) samples for water in the PC bottle, spiked at $5 \mu \mathrm{g} / \mathrm{L}$ and $10 \mu \mathrm{g} / \mathrm{L}$, (b) samples for tap water, spiked at $5 \mu \mathrm{g} / \mathrm{L}$ and $10 \mu \mathrm{g} / \mathrm{L}$. Peaks: (1) bisphenol A (2) ethynylestradiol, (3) diethylstilbestrol, (4) hexestrol, and (5) estrone. Other conditions are the same as in Figure 7.

method of HPLC-FLD-UV [38]. It should be noted that the highest EF values were achieved, compared with other methods based on different extraction materials. Moreover, the extraction time could be finished within $32 \mathrm{~min}$ for our method, which was significantly shorter than in other methods. These comparisons support the fact that this method is an effective analytical method for the determination of estrogen in environmental samples.

To confirm the applicability of the analytical method, two real samples were selected, including water in a PC bottle immersed in boiled water and tap water taken from our laboratory. Generally, exposure to BPA occurs mainly by consumption of contaminated foods and beverages that have contacted epoxy resins or PC plastics [8]. To test the hypothesis that bioactive BPA was released from PC bottles containing water and other beverages, the pretreatment and determination of BPA migrated from a polycarbonate bottle was attempted using the developed method. As shown in Table 4 and Figure 8, BPA was quantitatively detected in the water sample in the PC cup with a concentration of $8.3 \mu \mathrm{g} / \mathrm{L}$, although no compounds were detected in the tap water. The spike recoveries of the analytes were examined by the 
standard addition method. The concentration of addition in the sample was 0,5 , and $10 \mu \mathrm{g} / \mathrm{L}$. The recoveries were $75.0 \%-$ $121.1 \%$ for the ultrapure water in a PC bottle and $74.6 \%-$ $120.7 \%$ for the tap water, respectively. The abovementioned results indicated that this method could be applied to analyze trace estrogens in real samples.

\section{Conclusions}

In this study, aramid fiber was carbonized at an optimal temperature and characterized by SEM, Raman, and XPS, respectively. It was filled in a stainless-steel tube as a novel extraction material and connected to HPLC for use in an online in-tube SPME-HPLC system to detect five estrogens in water samples. The carbonized aramid fiber exhibited good durability, chemical stability, sensitivity, and excellent extraction performance compared with the pristine aramid fiber. Compared with the reported method for estrogen determination, this method was sensitive, fast, and had a high enrichment factor. Also, it was successfully applied to separate five estrogens and detect BPA in real samples with high extraction efficiency, good repeatability, and satisfied recoveries.

\section{Data Availability}

All data included in this study are available upon request by contact with the corresponding author.

\section{Conflicts of Interest}

The authors declare no conflicts of interest.

\section{Acknowledgments}

The research was supported by the National Natural Science Foundation of China (No. 22074029), the Key Projects of Science and Technology of Henan Province (Nos. $172102210085192102310262)$, the Science and Technology Project of Henan Province (No. 142102210047), and the Innovation Team in Henan Province (No. C20150020).

\section{References}

[1] Y. Corrotea, N. Aguilera, L. Honda, and P. Richter, "Determination of hormones in wastewater using rotating disk sorptive extraction and gas chromatography-mass spectrometry," Analytical Letters, vol. 49, no. 9, pp. 1344-1358, 2015.

[2] D. Bila, A. F. Montalvão, D. D. A. Azevedo, and M. Dezotti, "Estrogenic activity removal of $17 \beta$-estradiol by ozonation and identification of by-products," Chemosphere, vol. 69, no. 5, pp. 736-746, 2007.

[3] H. Kataoka, M. Ise, and S. Narimatsu, "Automated on-line intube solid-phase microextraction coupled with high performance liquid chromatography for the analysis of bisphenol A, alkylphenols, and phthalate esters in foods contacted with plastics," Journal of Separation Science, vol. 25, no. 1-2, pp. 77-85, 2002.

[4] F. Qiu, M. L. Peng, Z. M. Wei, X. J. Wang, and J. Yang, "Preparation of polyethersulfone/sulfonated polyethersulfonephenylethane microspheres and its application for the adsorption of bisphenol A," Journal of Applied Polymer Science, vol. 133, Article ID 4306, 2016.

[5] L. Honda, M. Becerra-Herrera, and P. Richter, "Liquid chromatography-time-of-flight high-resolution mass spectrometry study and determination of the dansylated products of estrogens and their hydroxylated metabolites in water and wastewater," Analytical and Bioanalytical Chemistry, vol. 410, no. 30, pp. 7909-7919, 2018.

[6] K. Kozlowska-Tylingo, J. Namieśnik, and T. Górecki, "Determination of estrogenic endocrine disruptors in environmental samples-a review of chromatographic methods," Critical Reviews in Analytical Chemistry, vol. 40, no. 3, pp. 194-201, 2010.

[7] Y. Wen, B.-S. Zhou, Y. Xu, S.-W. Jin, and Y.-Q. Feng, "Analysis of estrogens in environmental waters using polymer monolith in-polyether ether ketone tube solid-phase microextraction combined with high-performance liquid chromatography," Journal of Chromatography A, vol. 1133, no. 1-2, pp. 21-28, 2006.

[8] H. H. Le, E. M. Carlson, J. P. Chua, and S. M. Belcher, "Bisphenol A is released from polycarbonate drinking bottles and mimics the neurotoxic actions of estrogen in developing cerebellar neurons," Toxicology Letters, vol. 176, no. 2, pp. 149-156, 2008.

[9] H. Kataoka, A. Ishizaki, and K. Saito, "Recent progress in solid-phase microextraction and its pharmaceutical and biomedical applications," Analytical Methods, vol. 8, no. 29, pp. 5773-5788, 2016.

[10] M. Vinícius, M. Cestari, S. M. Palácio, S. D. De Campos, E. C. Muniz, and E. De Campos, "Silk fibroin nanofibers electrospun on glass fiber as a potential device for solid phase microextraction," Journal of Applied Polymer Science, vol. 132, Article ID 41717, 2015.

[11] E. Robotti, F. Campo, M. Riviello et al., "Optimization of the extraction of the volatile fraction from honey samples by SPME-GC-MS, experimental design, and multivariate target functions," Journal of Chemistry, vol. 2017, Article ID 6437857, 14 pages, 2017.

[12] P. Aberoomand Azar, S. Mohammadiazar, M. Saber Tehrani, and J. Norooz Haghi, "Nanoparticle-incorporated PDMS film as an improved performance SPME fiber for analysis of volatile components of Eucalyptus leaf," Journal of Chemistry, vol. 2013, Article ID 347927, 6 pages, 2013.

[13] A. R. Mansur, T. G. Nam, H. W. Jang et al., "Determination of 2-propenal using headspace solid-phase microextraction coupled to gas chromatography-time-of-flight mass spectrometry as a marker for authentication of unrefined sesame oil," Journal of Chemistry, vol. 2017, Article ID 9106409, 12 pages, 2017.

[14] H. L. Lord and J. Pawliszyn, "Method optimization for the analysis of amphetamines in urine by solid-phase microextraction," Analytical Chemistry, vol. 69, no. 19, pp. 3899-3906, 1997.

[15] H. Kataoka, "SPME techniques for biomedical analysis," Bioanalysis, vol. 7, no. 17, pp. 2135-2144, 2015.

[16] M. Fernández-Amado, M. C. Prieto-Blanco, P. López-Mahía, S. Muniategui-Lorenzo, and D. Prada-Rodríguez, "Strengths and weaknesses of in-tube solid-phase microextraction: a scoping review," Analytica Chimica Acta, vol. 906, pp. 41-57, 2016.

[17] M. He, B. Chen, and B. Hu, "Recent developments in stir bar sorptive extraction," Analytical and Bioanalytical Chemistry, vol. 406, no. 8, pp. 2001-2026, 2013. 
[18] N. Ibrahim, R. Osman, A. Abdullah, and N. Saim, "Determination of phthalate plasticisers in palm oil using online solid phase extraction-liquid chromatography (SPE-LC)," Journal of Chemistry, vol. 2014, Article ID 682975, 9 pages, 2014.

[19] M. C. Prieto-Blanco, C. Cháfer-Pericás, P. López-Mahía, and P. Campíns-Falcó, "Automated on-line in-tube solid-phase microextraction-assisted derivatization coupled to liquid chromatography for quantifying residual dimethylamine in cationic polymers," Journal of Chromatography A, vol. 1188, no. 2, pp. 118-123, 2008.

[20] H. Kataoka, "Recent developments and applications of microextraction techniques in drug analysis," Analytical and Bioanalytical Chemistry, vol. 396, no. 1, pp. 339-364, 2010.

[21] Y. Yamamoto, A. Ishizaki, H. Kataoka, and J. Chromatogr, "Biomonitoring method for the determination of polycyclic aromatic hydrocarbons in hair by online in-tube solid-phase microextraction coupled with high performance liquid chromatography and fluorescence detection," Journal of Chromatography B, vol. 1000, pp. 187-191, 2015.

[22] J. Liu, W. Zhao, S. Li, A. Zhang, Y. Zhang, and S. Liu, "Determination of volatile compounds in foxtail millet sake using headspace solid-phase microextraction and gas chromatography-mass spectrometry," Journal of Chemistry, vol. 2015, Article ID 239016, 9 pages, 2015.

[23] R. Eisert and J. Pawliszyn, "Automated in-tube solid-phase microextraction coupled to high-performance liquid chromatography," Analytical Chemistry, vol. 69, no. 16, pp. 3140-3147, 1997.

[24] Y. Hu, C. Song, and G. Li, "Fiber-in-tube solid-phase microextraction with molecularly imprinted coating for sensitive analysis of antibiotic drugs by high performance liquid chromatography," Journal of Chromatography A, vol. 1263, pp. 21-27, 2012.

[25] M. E. C. Queiroz and L. P. Melo, "Selective capillary coating materials for in-tube solid-phase microextraction coupled to liquid chromatography to determine drugs and biomarkers in biological samples: a review," Analytica Chimica Acta, vol. 826, pp. 1-11, 2014.

[26] M. C. Prieto-Blanco, S. Peñafiel Barba, Y. Moliner-Martínez, and P. Campíns-Falcó, "Footprint of carbonyl compounds in hand scent by in-tube solid-phase microextraction coupled to nano-liquid chromatography/diode array detection," Journal of Chromatography A, vol. 1596, pp. 241-249, 2019.

[27] J. Feng, M. Sun, S. Han et al., "Polydopamine-coated cotton fibers as the adsorbent for in-tube solid-phase microextraction," Journal of Separation Science, vol. 42, no. 12, pp. 2163-2170, 2019.

[28] X. Wang, J. Feng, J. Feng, Y. Tian, C. Luo, and M. Sun, "Basalt fibers coated with nano-calcium carbonate for in-tube solidphase microextraction and online analysis of estrogens coupled with high-performance liquid chromatography," Analytical Methods, vol. 10, no. 19, pp. 2234-2241, 2018.

[29] C. Li, M. Sun, X. Ji et al., "Carbonized cotton fibers via a facile method for highly sensitive solid-phase microextraction of polycyclic aromatic hydrocarbons," Journal of Separation Science, vol. 42, no. 12, pp. 2155-2162, 2019.

[30] X. Ji, M. Sun, C. Li et al., "Bare polyprolylene hollow fiber as extractive phase for in-tube solid-phase microextraction to determine estrogens in water samples," Journal of Separation Science, vol. 42, no. 14, pp. 2398-2406, 2019.

[31] C. Jia, R. Zhang, C. Yuan et al., "Surface modification of aramid fibers by amino functionalized silane grafting to improve interfacial property of aramid fibers reinforced composite," Polymer Composites, vol. 41, no. 5, pp. 2046-2053, 2020.

[32] L. Liu, Y. D. Huang, Z. Q. Zhang, B. Jiang, and J. Nie, "Ultrasonic modification of aramid fiber-epoxy interface," Journal of Applied Polymer Science, vol. 81, no. 11, pp. 2764-2768, 2001.

[33] W. Qi, M. Q. Jian, C. Y. Wang, and Y. J. Zhang, "Carbonized silk nanofiber membrane for transparent and sensitive electronic skin," Advanced Functional Materials, vol. 27, Article ID 1605657, 2017.

[34] J. Feng, M. Sun, Y. Bu, and C. Luo, "Hollow fiber membranecoated functionalized polymeric ionic liquid capsules for direct analysis of estrogens in milk samples," Analytical and Bioanalytical Chemistry, vol. 408, no. 6, pp. 1679-1685, 2016.

[35] Y. Hu, Y. Wang, X. Chen, Y. Hu, and G. Li, "A novel molecularly imprinted solid-phase microextraction fiber coupled with high performance liquid chromatography for analysis of trace estrogens in fishery samples," Talanta, vol. 80, no. 5, pp. 2099-2105, 2010.

[36] L. Chen, M. Mei, X. Huang, and D. Yuan, "Sensitive determination of estrogens in environmental waters treated with polymeric ionic liquid-based stir cake sorptive extraction and liquid chromatographic analysis," Talanta, vol. 152, pp. 98104, 2016.

[37] Y. Zou, Y. Li, H. Jin et al., "Determination of estrogens in human urine by high-performance liquid chromatography/ diode array detection with ultrasound-assisted cloud-point extraction," Analytical Biochemistry, vol. 421, no. 2, pp. 378-384, 2012.

[38] X. Luo, G. Li, and Y. Hu, "In-tube solid-phase microextraction based on NH 2-MIL-53 (Al)-polymer monolithic column for online coupling with high-performance liquid chromatography for directly sensitive analysis of estrogens in human urine," Talanta, vol. 165, pp. 377-383, 2017.

[39] R. Sadowski and R. Gadzała-Kopciuch, "Isolation and determination of estrogens in water samples by solid-phase extraction using molecularly imprinted polymers and HPLC," Journal of Separation Science, vol. 36, no. 14, pp. 2299-2305, 2013. 